\title{
Ansiedade estado pré-competitiva em atletas de voleibol infanto-juvenis
}

CDD. 20.ed. 796.011

796.325

http://dx.doi.org/10.1590/1807-55092016000401061

\author{
Thais do Amaral MACHADO* \\ Mayara Juliana PAES* \\ Shelse Marinho ALQUIERE* \\ Ana Claudia Vecchi OSIECKI* \\ Luciana da Silva LIRANI* \\ Joice Mara Facco STEFANELLO*
}

*Setor de Ciências Biológicas, Universidade Federal do Paraná, $\mathrm{PR}$, Brasil.

\section{Resumo}

Compreender a relação entre ansiedade-estado pré-competitiva e desempenho esportivo se constitui em importante meio para ajudar os esportistas a diagnosticar e controlar emoções. 0 presente estudo pretendeu: a) comparar o nivel de ansiedade pré-competitiva entre atletas homens e mulheres, das equipes finalistas da Taça Paraná de Voleibol 2011; b) comparar os níveis de ansiedade pré-competitiva entre as equipes campeã e vice-campeã masculina, bem como entre as equipes campeã e vice-campeã feminina; c) comparar os niveis de ansiedade pré-competitiva dos atletas titulares e reservas, para as equipes de ambos os sexos; d) verificar a relação entre os níveis de ansiedade pré-competitiva e o tempo de prática dos atletas das equipes masculinas, bem como das equipes femininas. Participaram do estudo quatro equipes infantojuvenis, finalistas da Taça Paraná 2011, totalizando 24 atletas do sexo feminino e 23 do sexo masculino (idade 16,16 0,34 anos) e tempo de prática (4,97 \pm 1,99 anos). Para avaliar a ansiedade pré-competitiva, utilizou-se o instrumento CSAI-2. As coletas ocorreram 30 minutos antes do início dos jogos finais. De forma geral, as equipes finalistas apresentaram niveis de ansiedade geral pré-competitiva similares, embora tenham sido encontradas diferenças significativas entre atletas mulheres titulares e reservas em relação à autoconfiança, sendo que as titulares apresentaram médias mais elevadas neste aspecto.

Palavras-Chave: Psicologia do esporte; Competição; Avaliação; CSAI-2.

\section{Introdução}

O ambiente esportivo competitivo é multifacetado, compreendendo situaçóes que dizem respeito ao jogo, aos sentimentos dos atletas e aos acontecimentos extraquadra, como torcida, família, pressão dos técnicos, entre outros. Tais situaçôes despertam diversas reaçóes emocionais nos atletas, que tanto podem auxiliar como prejudicar o seu desempenho ${ }^{1}$.

Uma das reaçóes emocionais de maior relevância para a atuação esportiva é a ansiedade, vista como um estado subjetivo de inquietaçáo ou desordem, decorrente de uma situação antecipada de ameaça real ou imaginária. Pode ser considerada como uma característica estável da personalidade (ansiedade traço), el ou com um estado emocional transitório (ansiedade-estado), que aparece em momentos específicos ${ }^{1}$.
As sensaçôes corporais desagradáveis ${ }^{2}$ que acompanham a ansiedade podem interferir no comportamento dos atletas em momentos de competição, gerando reaçôes psicológicas e fisiológicas que tendem a prejudicar seu rendimento ${ }^{3}$. Entre os sinais psicológicos, encontram-se fatores como irritabilidade, confusão, diminuição da concentração, insegurança e instabilidade de humor. No âmbito das alteraçôes fisiológicas, constatam-se reaçôes como aumento da frequência cardíaca, respiratória e da circulação sanguínea, além de maior sudorese, tremores e tensão muscular ${ }^{4}$. É comum que esses sinais se façam presentes nos atletas antes de partidas e provas, principalmente, se o atleta sente-se inseguro em relação às suas capacidades perante os 
adversários, e incerto no que diz respeito ao resultado da competiçãa 5 .

Estudo prévio com atletas jovens de modalidades coletivas e individuais apontou diferenças na ansiedade pré-competitiva relacionadas ao sexo, nível de competição, personalidade do atleta, tempo de experiência e esporte praticado ${ }^{1}$. Em modalidades como o voleibol, a ansiedade tem sido associada com o desempenho pré-competitivo e competitivo dos atletas, mostrando que a ansiedade-estado cognitiva pode prejudicar o desempenho esportivo de atletas femininas de categorias infantis ${ }^{6}$. Além disso, com equipes adultas de alto rendimento, tem-se encontrado relação da ansiedade com a experiência na modalidade praticada, a posiçáo em quadra e o rendimento máximo sob pressão. Atletas de voleibol com maior tempo de experiência obtiveram pontuaçôes mais elevadas em competências psicológicas gerais, recursos pessoais de confronto, melhor concentração e rendimento máximo ${ }^{7}$.

Observa-se que a ansiedade-estado pode se apresentar em maiores níveis no período em que antecede a competição, podendo diminuir no decorrer da mesma e também tendo chances de elevar-se ao término do campeonato ${ }^{8}$. Em atletas jovens, essas reaçóes podem ser influenciadas por diferentes fatores inerentes a idade, situaçôes e experiências vivenciadas neste período, além do nível da competiçâo, e se intensificar em momentos decisivos, como a final de um campeonato?

\section{Método}

Participaram do estudo quatro equipes infanto-juvenis finalistas da Taça Paraná de Voleibol 2011 (média de idade geral 16,16 $\pm 0,34$ anos), totalizando 24 atletas do sexo feminino e 23 do sexo masculino. Todos os atletas finalistas apresentaram tempo de prática de 4,97 $\pm 1,99$ anos na modalidade voleibol e já haviam disputado competiçôes importantes a nível estadual e nacional. Todos os atletas responderam ao questionário individualmente no vestiário 30 minutos antes do início do aquecimento para os jogos das finais, aplicado por uma das pesquisadoras. As informaçóes descritivas da amostra referentes ao tempo de prática na modalidade, idade e se haviam disputado competiçôes estaduais e/ou nacionais foram informadas no cabeçalho do instrumento CSAI-2.

O estudo foi aprovado pelo Comitê de Ética em Pesquisa do Hospital de Clínicas da Universidade Federal do Paraná, atendendo a Resolução 196/96, sob o registro: CEP/SD: 9750; CAAE: 01309012.7.0000.0096.
Tendo em vista que a ansiedade pode afetar o rendimento esportivo, faz-se necessário investigar os sintomas e manifestações que podem interferir em atletas na fase da adolescência, que corresponde a uma fase de mudanças significativas e relevantes acerca do seu futuro ${ }^{6}$. Desta forma, investigaçôes sobre a ansiedade-estado pré-competitiva de atletas de voleibol finalistas de uma competição de categoria de base com ampla representatividade no cenário Sul Americano, se constitui em um meio relevante no intuito de contribuir na área de conhecimento e auxiliar esportistas, técnicos e profissionais a diagnosticarem e controlarem suas emoçóes, buscando uma atuação eficaz, por meio de programas de treinamento de competências psicológicas voltadas ao esporte.

O presente estudo pretendeu: a) comparar o nível de ansiedade pré-competitiva entre os atletas homens e mulheres, das equipes finalistas da Taça Paraná de Voleibol 2011; b) comparar os níveis de ansiedade pré-competitiva entre as equipes campeã e vice-campeã masculina, bem como entre as equipes campeã e vice-campeã feminina; c) comparar os níveis de ansiedade pré-competitiva dos atletas titulares e reservas, para as equipes de ambos os sexos; d) verificar a relação entre os níveis de ansiedade pré-competitiva e o tempo de prática dos atletas das equipes masculinas, bem como das equipes femininas.

Para avaliar a ansiedade pré-competitiva, utilizou-se o Questionário de Ansiedade Competitiva (CompetitiveStateAnxiety Inventory-2 - CSAI-2) ${ }^{10}$; validado para língua portuguesa por CRUZ et al. ${ }^{11}$. O CSAI-2 é um instrumento de medida multidimensional, específico para o esporte, utilizado para diagnosticar e qualificar o nível de ansiedade (somática e cognitiva) e a ansiedade relacionada à autoconfiança. É composto por 27 questôes divididas em três dimensões: ansiedade cognitiva, ansiedade somática e autoconfiança, com nove itens em cada, para as quais o atleta opta por $1=$ nada; $2=$ um pouco; $3=$ moderado; $4=$ muito. A pontuação total é obtida pela somatória das respostas de cada dimensão, variando entre 9 (baixa ansiedade) a 36 (alta ansiedade) pontos ${ }^{11}$.

Para o tratamento dos dados foi utilizado o teste Shapiro Wilk, que demonstrou a distribuição normal dos dados. Em seguida, utilizou-se o teste 
t de Student a fim de verificar as diferenças para a ansiedade geral e suas dimensóes (ansiedade cognitiva, ansiedade somática e autoconfiança) entre os atletas homens e mulheres, campeóes e vice-campeões e titulares e reservas. A correlação de Spearman foi utilizada para analisar a relação entre ansiedade pré-competitiva e o tempo de prática. Para todas as análises considerou-se nível de significância de 5\%.

O tamanho do efeito e o poder estatístico foram calculados por meio do "software" G-Power. Quanto ao poder do teste, este está relacionado ao número de sujeitos adequados às análises. Assim, como se trata de equipes de voleibol ao qual envolvem o número máximo de 12 atletas relacionados por jogo e considerando que o objetivo do estudo envolve apenas as equipes finalistas da competição, o número de indivíduos avaliados foi restrito à campeã e vice-campeã masculino e feminino (quatro equipes).
Embora o tamanho do efeito nos testes realizados se mostre pequeno, é importante ressaltar que a presente pesquisa investigou atletas das melhores equipes do cenário nacional nesta categoria e possui caráter exploratório apresentando achados preliminares sobre a temática (voleibol) e a população estudada (atletas infanto-juvenis) tornando-se relevante em relação ao respectivo contexto. Também foi verificado que em estudos prévios ${ }^{12-13}$ náo foi apresentado o poder das análises e o tamanho do efeito, tornando esta investigação mais clara quanto os parâmetros das análises realizadas.

A TABELA 1 apresenta os dados referentes à idade e tempo de prática das equipes campeã e vice-campeã masculina e feminina.

A TABELA 1 apresenta a média de idade e o tempo de prática dos atletas das quatro equipes finalistas da competição, as quais não se diferem entre os gêneros. De modo geral, os atletas são jovens e com tempo de prática 4,97 $\pm 1,99$ anos.

TABELA 1 - Média de idade e tempo de prática dos atletas campeões e vice-campeões masculinos e femininos.

\begin{tabular}{lcc}
\hline Equipes & Idade & Tempo de prática \\
\hline Campeã masculina & $16,16 \pm 0,57$ & $6,20 \pm 1,43$ \\
Vice- campeã masculina & $16,63 \pm 0,50$ & $4,45 \pm 1,91$ \\
Campeã feminina & $15,91 \pm 0,79$ & $5,08 \pm 2,35$ \\
Vice-campeã feminina & $15,91 \pm 0,90$ & $4,08 \pm 1,67$ \\
\hline
\end{tabular}

\section{Resultados}

A TABELA 2 apresenta os resultados do teste $t$ de Student realizado entre atletas homens e mulheres finalistas da Taça Paraná de Voleibol, no que diz respeito à ansiedade geral pré-competitiva e suas dimensóes (ansiedade cognitiva, ansiedade somática e autoconfiança).

TABELA 2 - Comparação da ansiedade geral, dimensões do CSAI-2 (ansiedade cognitiva, ansiedade somática e autoconfiança) entre atletas de voleibol homens e mulheres.

\begin{tabular}{lcccccc}
\hline \multirow{2}{*}{ Variáveis } & \multicolumn{2}{c}{ Homens } & \multicolumn{2}{c}{ Mulheres } & p & Poder da análise \\
& $\mathbf{M}$ & DP & M & DP & & \\
\hline Ansiedade cognitiva & 20,17 & $\pm 4,01$ & 21,00 & $\pm 4,63$ & 0,51 & 0,15 \\
Ansiedade somática & 15,56 & $\pm 3,32$ & 16,00 & $\pm 5,63$ & 0,74 & 0,08 \\
Autoconfiança & 27,26 & $\pm 5,46$ & 26,62 & $\pm 5,72$ & 0,69 & 0,10 \\
Ansiedade geral & 63,08 & $\pm 5,50$ & 63,5 & $\pm 7,87$ & 0,83 & 0,06 \\
\hline
\end{tabular}

Não foram encontradas diferenças estatisticamente significativas entre homens e mulheres para a ansiedade pré-competitiva geral, bem como para as dimensôes ansiedade cognitiva, ansiedade somática, autoconfiança.
A TABELA 3 apresenta os resultados da comparação dos níveis de ansiedade pré-competitiva, bem como das dimensões ansiedade cognitiva, somática e autoconfiança entre as equipes campeã e vice-campeã masculinas e femininas. 
TABELA 3 - Comparação da ansiedade geral, dimensões do CSAI-2 (ansiedade cognitiva, ansiedade somática e autoconfiança) entre atletas homens e mulheres campeões e vice-campeões.

\begin{tabular}{|c|c|c|c|c|c|c|c|c|c|c|c|c|}
\hline \multirow[t]{2}{*}{ Variáveis } & \multicolumn{2}{|c|}{$\begin{array}{c}\text { Equipe } \\
\text { Campeá Masc. }\end{array}$} & \multicolumn{2}{|c|}{$\begin{array}{l}\text { Equipe Vice- } \\
\text { Campeá Masc. }\end{array}$} & \multirow{2}{*}{$\mathbf{p}$} & \multirow[t]{2}{*}{ Poder } & \multicolumn{2}{|c|}{$\begin{array}{c}\text { Equipe } \\
\text { Campeã Fem. }\end{array}$} & \multicolumn{2}{|c|}{$\begin{array}{l}\text { Equipe Vice- } \\
\text { Campeã Fem. }\end{array}$} & \multirow{2}{*}{$\mathbf{p}$} & \multirow[t]{2}{*}{ Poder } \\
\hline & $\mathbf{M}$ & DP & $\mathbf{M}$ & DP & & & $\mathbf{M}$ & DP & $\mathbf{M}$ & DP & & \\
\hline $\begin{array}{l}\text { Ansiedade } \\
\text { cognitiva }\end{array}$ & 19,5 & $\pm 4,14$ & 4,45 & $\pm 1,91$ & 0,41 & 0,20 & 21,66 & $\pm 4,31$ & 20,33 & $\pm 5,03$ & 0,49 & 0,17 \\
\hline $\begin{array}{l}\text { Ansiedade } \\
\text { somática }\end{array}$ & 15,58 & $\pm 2,71$ & 15,54 & $\pm 4,03$ & 0,97 & 0,04 & 16,16 & $\pm 5,30$ & 15,83 & $\pm 6,22$ & 0,88 & 0,05 \\
\hline Autoconfiança & 28,33 & $\pm 5,54$ & 26,09 & $\pm 5,37$ & 0,33 & 0,25 & 26,75 & $\pm 5,54$ & 26,50 & $\pm 6,14$ & 0,91 & 0,05 \\
\hline Ansiedade geral & 63,41 & $\pm 2,23$ & 62,72 & $\pm 7,81$ & 0,78 & 0,08 & 64,41 & $\pm 6,93$ & 62,58 & $\pm 8,93$ & 0,58 & 0,13 \\
\hline
\end{tabular}

Observa-se que não há diferenças estatisticamente significativas para atletas homens e mulheres, campeôes e vice-campeóes para a ansiedade pré-competitiva geral e as dimensóes ansiedade cognitiva, ansiedade somática e autoconfiança.
A TABELA 4 apresenta os resultados do teste $\mathrm{t}$ de Student realizado entre atletas titulares e reservas de cada uma das equipes (masculina e feminina), considerando a ansiedade geral pré-competitiva e as dimensóes ansiedade cognitiva, ansiedade somática e autoconfiança.

TABELA 4 - Comparação da ansiedade geral e dimensões do CSAI-2 (ansiedade cognitiva, ansiedade somática e autoconfiança) entre titulares e reservas homens e mulheres.

$p<0,05^{*}$

Efect Size Autoconfiança feminina Cohens' d $=0,87$.

\begin{tabular}{|c|c|c|c|c|c|c|c|c|c|c|c|c|}
\hline \multirow{2}{*}{ Variáveis } & \multicolumn{2}{|c|}{ Titulares } & \multicolumn{2}{|c|}{ Reservas } & \multirow[b]{2}{*}{$\mathbf{p}$} & \multirow{2}{*}{ Poder } & \multicolumn{2}{|c|}{ Titulares } & \multicolumn{2}{|c|}{ Reservas } & \multirow[b]{2}{*}{$\mathbf{p}$} & \multirow{2}{*}{ Poder } \\
\hline & $\mathbf{M}$ & DP & $\mathbf{M}$ & DP & & & $\mathbf{M}$ & DP & $\mathbf{M}$ & DP & & \\
\hline $\begin{array}{l}\text { Ansiedade } \\
\text { cognitiva }\end{array}$ & 19,64 & $\pm 19,64$ & 21,00 & $\pm 3,87$ & 0,43 & 0,19 & 20,21 & $\pm 4,49$ & 22,10 & $\pm 4,84$ & 0,34 & 0,25 \\
\hline $\begin{array}{l}\text { Ansiedade } \\
\text { somática }\end{array}$ & 15,78 & $\pm 3,87$ & 15,22 & $\pm 2,81$ & 0,68 & 0,10 & 16,07 & $\pm 5,31$ & 15,90 & $\pm 6,40$ & 0,94 & 0,04 \\
\hline Autoconfiança & 27,50 & $\pm 5,85$ & 26,88 & $\pm 5,10$ & 0,79 & 0,07 & 28,57 & $\pm 5,43$ & 23,90 & $\pm 5,19$ & $0,04^{*}$ & 0,68 \\
\hline Ansiedade geral & 62,92 & $\pm 6,63$ & 63,33 & $\pm 3,42$ & 0,84 & 0,06 & 64,71 & $\pm 7,49$ & 61,80 & $\pm 8,48$ & 0,39 & 0,22 \\
\hline
\end{tabular}

Observa-se diferença significativa $(\mathrm{p}=0,04)$ quando comparadas as atletas mulheres titulares e reservas para a dimensão autoconfiança. $\mathrm{O}$ tamanho do efeito para esta análise foi de Cohens' $\mathrm{d}=0,87$, classificado como grande, apontando que para esta dimensão o tamanho reduzido da amostra parece não afetar o resultado, sendo que em pequenas amostras, mas com grandes efeitos, entende-se que os aspectos estudados tem grande impacto. No entanto, não foram encontradas diferenças estatisticamente significativas para os demais aspectos da ansiedade pré-competitiva geral, bem como para as dimensôes ansiedade cognitiva e ansiedade somática em relação à titularidade.

A TABELA 5 apresenta os valores de média e desvio padrão da ansiedade geral e tempo de prática de homens e mulheres, bem como o valor da relação entre estas variáveis.

Percebe-se que não houve relação estatisticamente significativa entre as variáveis ansiedade geral e tempo de prática para atletas homens e mulheres finalistas da competição.

TABELA 5 - Correlação da ansiedade geral e o tempo de prática para atletas homens e atletas mulheres.

$p<0,05^{*}$

\begin{tabular}{lcccccc}
\hline & \multicolumn{2}{c}{ Ansiedade geral } & \multicolumn{2}{c}{ Tempo de prática } & p & r \\
& M & DP & M & DP & & \\
\hline Homens & 63,09 & 5,51 & 4,58 & 2,06 & 0,43 & 0,17 \\
Mulheres & 63,5 & 7,88 & 5,37 & 1,87 & 0,46 & 0,16 \\
\hline
\end{tabular}




\section{Discussão}

A partir da análise dos dados, observa-se que não foram encontradas diferenças estatisticamente significativas entre homens e mulheres para a ansiedade pré-competitiva geral, bem como para as dimensóes ansiedade cognitiva e ansiedade somática, na dimensão autoconfiança encontrou-se diferenças significativas entre atletas mulheres titulares e reservas. Em estudo realizado com atletas amadores de natação da mesma faixa etária e de ambos os sexos em fase final de competição, o qual avaliou a ansiedade estado, por meio do instrumento CSAI-2 observou que as meninas e os atletas com menor experiência competitiva apresentaram maiores níveis de ansiedade cognitiva e somática e diferença na autoconfiança entre atletas de maior e menor experiência competitiva ${ }^{14}$.

Quando detalhado o resultado em relação às dimensôes do instrumento, nota-se que os atletas do sexo masculino demonstraram níveis mais elevados de autoconfiança do que as atletas femininas. Diferente dos resultados encontrados no presente estudo, com atletas de natação, que participaram de duas competições, estaduais e regionais, utilizando-se também do instrumento CSAI-2 para avaliar a ansiedade pré-competitiva, observou-se que as mulheres apresentaram tendência de serem mais ansiogênicas em relação aos homens ${ }^{12}$. Esse fato pode ter ocorrido devido à percepção de autoconfiança que influencia a conduta do atleta, que se percebe capaz de executar as tarefas necessárias para sua prática esportiva ${ }^{15-16}$. Os atletas do sexo masculino tendem a se autoavaliar a respeito de suas capacidades de maneira mais positiva em relação às mulheres atletas, como observado no presente estudo ${ }^{17}$.

Também entre as equipes campeã e vice-campeã feminina não foram encontradas diferenças estatisticamente significativas para a ansiedade pré-competitiva geral, bem como para as dimensóes ansiedade cognitiva, ansiedade somática e autoconfiança. Já com atletas jovens de tênis, de ambos os sexos, durante um torneio, avaliou-se a ansiedade dos esportistas pelo instrumento CSAI-2 e encontrou-se que as mulheres apresentaram maiores níveis de ansiedade somática em relação aos homens e os campeóes apresentaram menor ansiedade cognitiva e maiores escores de autoconfiança em relaçáo aos perdedores e a ansiedade somática foi menor nos perdedores ${ }^{18}$, o que diverge dos achados encontrados no presente estudo. Contudo, estas diferenças podem estar associadas às características cognitivas específicas dos esportes coletivos e individuais que merecem ser mais bem investigados.
Por outro lado, no presente estudo, encontraram-se diferenças estatisticamente significativas entre atletas mulheres titulares e reservas no que diz respeito à dimensão autoconfiança, sendo que as titulares apresentaram maior média neste aspecto $p$ $\leq 0,05$, além do poder do teste $(0,68)$ considerado médio e o tamanho do efeito grande $\mathrm{d}=0,87^{19}$. Em estudo com atletas do sexo masculino de futsal, com a mesma faixa etária, foram avaliadas diferenças entre o tipo de ansiedade apresentada entre jogadores titulares e reservas, sendo que também se notaram médias mais elevadas na dimensão autoconfiança para atletas titulares e reservas, porém não se observaram diferenças significativas entre os grupos (titular e reserva). Além disso, os titulares apresentaram maior ansiedade somática, enquanto os reservas apresentaram maior ansiedade cogniti$\mathrm{va}^{13}$. A divergência nestes achados demonstra que a titularidade dos atletas parece não ser um fator determinante da ansiedade pré-competitiva. A diferença na autoconfiança pode ser explicada pelo momento importante da competição, o qual exige das atletas maior confiança em suas capacidades e desempenho. As atletas titulares podem apresentar maior autoconfiança que as atletas reservas devido ao fato de terem sido escaladas para a partida previamente, tendo a confirmação de sua participação em quadra, diferentemente das atletas reservas, que ficam incertas quanto sua presença em quadra nesta partida e ao longo da competição ${ }^{13}$.

Quanto ao tempo de prática, não se observou correlação significativa com o nível de ansiedade pré-competitiva para homens e mulheres, campeóes e vice-campeóes. Em investigação prévia com atletas de natação juvenis, de ambos os sexos, os nadadores menos experientes apresentaram menores médias na dimensão autoconfiança em relação aos mais experientes ${ }^{14}$. Este achado pode estar relacionado à variaçáo da ansiedade de acordo com a idade, sendo observados maiores níveis ao fim da adolescência, tanto para homens como para as mulheres ${ }^{8}$. Os homens tendem a participar de atividades com grau mais elevado de exigência, o que pode afetar os níveis de ansiedade. No entanto, no presente estudo não se observou diferenças entre os sexos. Porém, é necessário ainda maior aprofundamento em relaçáo a este aspecto para ambos os sexos, sendo possível, a partir de uma amostra maior, realizar inferências a respeito destas variáveis. Visto que pesquisas com mulheres ainda são relativamente menores em 
relação às pesquisas com os homens na questão da ansiedade ${ }^{20}$. Além disso, a realização de estudos qualitativos para investigaçáo e aprofundamento das fontes de ansiedade, assim como o estudo realizado com treinadores esportivos ${ }^{21}$, é importante para um melhor entendimento sobre aspectos psicológicos que interferem no desempenho dos atletas.

Observa-se, principalmente, que as equipes finalistas demonstraram níveis de ansiedade geral parecidas, o que pode ter ocorrido devido ao momento da competição, por se tratar justamente de partidas decisivas para se consagrarem campeóes da competição.

Com relação ao número de atletas analisados, sugere-se maiores investigaçóes agregando dados de finalistas de outras categorias e competiçóes, já que o poder estatístico encontrado nas análises foi baixo, sugerindo que o tamanho da amostra foi um limitador neste estudo. Da mesma forma, com o intuito de expandir as comparações entre atletas dos naipes masculino e feminino, bem como titulares e reservas poderiam ser também realizadas com equipes que não chegaram as finais da competiçáo. Para estudos futuros, sugere-se contemplar a avaliação da intensidade, direção e frequência da ansiedade, visto que são fatores determinantes para compreensão de situaçóes que podem interferir no rendimento atlético, tendo em vista que trabalhos voltados a estes aspectos psicológicos podem auxiliar os atletas em situaçóes adversas em partidas ou ao longo de competiçóes.

\title{
Nota
}

As autoras participam do Programa de Pós Graduação em Educação Física do Departamento de Educação Física da Universidade Federal do Paraná, Curitiba, Paraná.

\begin{abstract}
Pre-competitive state anxiety in infant juvenile volleyball athletes

Understanding the relationship between pre-competitive state anxiety and sports performance are an important means to help sportsmen to diagnose and manage emotions. The present study aimed to: a) compare the level of pre-competitive anxiety among athletes men and women, the finalist teams of Paraná Cup Volleyball 2011 ; b) to compare the levels of pre-competitive anxiety among male champion teams and runners-up, as well as between female champion teams and runners-up; c) compare the levels of pre-competitive anxiety and reserves holders athletes for teams of both sexes; d) verify the relationship between the levels of pre-competitive anxiety and practice time for athletes of men's teams and the women's teams. Study participants were four youth teams, Paraná Cup finalists in 2011, totaling 24 female athletes and 23 male athletes (age $16.16 \pm$ overall 0.34 years) and practice time $4.97 \pm 1.99$ years. To evaluate the pre-competitive anxiety, used the instrument CSAI-2. Sample occurred 30 minutes before the start of finals games. In general, the finalist teams had higher levels of general pre-competitive anxiety similar, although statistically significant differences between women athletes holders and reserves have been found in relation to the self-confidence, and the holders had higher average in this aspect.
\end{abstract}

KEY WoRDS: Sport psychology; Competition; Evaluation; CSAI-2.

\section{Referências}

1. Gonçalves MP, Belo RP. Ansiedade-traço competitiva: diferenças quanto ao gênero, faixa etária, experiência em competiçóes e modalidade esportiva em jovens atletas. Psico-USF. 2007;12:301-7.

2. Raedeke TD, Focht BC, Scales D. Social environmental factors and psychological responses to acute exercise for socially physique anxious females. Psychol Sport Exerc. 2007;8:463-76. 
3. Stefanello JM. Ansiedade competitiva e os fatores de personalidade de adolescentes que praticam voleibol: um estudo causal-comparativo. Kinesis. 1990;6:203-24.

4. Lavoura NL, Botura HML, Machado AA. Estudo da ansiedade e as diferenças entre os gêneros em um esporte de aventura competitivo. Rev Bras Educ Fís Esporte Lazer Dança. 2006;1:74-81.

5. Feltz DL. Self-confidence, self-efficacy and anxiety. In: Bartlett R, Gratton C, Rolf C, editors. Encyclopedia of international sports studies. NewYork: Routledge; 2006. p.1180-1.

6. Sonoo CN, Gomes AL, Damasceno ML, Ribas S. Ansiedade e desempenho: um estudo com uma equipe infantil de voleibol feminino. Motriz. 2010;16:629-37.

7. Dinis LG. Habilidades psicológicas e ansiedade traço em voleibol. Coimbra: Universidade de Coimbra; 2003.

8. Cratty BJ. Psicologia no esporte: psicologia do esporte. Rio de Janeiro: Prentice Hall do Brasil; 1984.

9. Kitsantas A, Zimmerman B. Comparing self-regulatory processes among novice non-expert and expert volleyball players a microanalytic study. J Appl Sport Psychol. 2002;14:91-105.

10. Martens R, Burton D, Vealey R, Bump L, Smith D. The development and validation of the Competitive State Anxiety Inventory-2. In: Martens R, Vealey RS, Burton D. Competite anxiety in sport. Champaign: Human Kinetics; 1990. p.117-90.

11. Cruz JF, Viveiros MI, Alvez LA, et al. Características psicométricas de uma versão portuguesa do "Competitive State Anxiety Inventory-2” (CSAI-2): dados preliminares relativos à sua validade e estrutura factorial. VI Simpósio Nacional de Investigação em Psicologia: actas do Simpósio Nacional de Investigação em Psicologia; 28-30 nov 2006; Évora, PORT. Évora: Departamento de Psicologia da Universidade de Évora; 2006. vol.3, p.104-25.

12. Barbacena MM, Grisi RNF. Nível de ansiedade pré-competitiva em atletas de natação. Conexôes. 2008;6:31-9.

13. Bocchini D, Morimoto L, Rezende D, Cavinato G, Luz LMR. Análise dos tipos de ansiedade entre jogadores titulares e reservas de futsal. Conexóes. 2008;6 Espec:522-32.

14. Souza MAP, Teixeira RB, Lobato PL. Manifestação da ansiedade pré-competitiva em nadadores amadores. Rev Educ Fís/UEM. 2012;23:195-203.

15. Cruz J, Vianna M. O treino das competências psicológicas e a preparação mental para a competição. In: Cruz J, editor. Manual de psicologia do desporto. Braga: Sistemas Humanos e Organizacionais; 1996. p.533-63.

16. Stefanello JMF. Treinamento de competências psicológicas em busca da excelência esportiva. Barueri: Manole; 2007.

17. Feltz DL, Short SE, Sullivan PJ. Self-efficacy in sport: reserch and strategies for working with athletes, teams, and coaches. Int J Sport Sci Coach. 2008;3:293-5.

18. Filaire E, Alix D, Ferrand C, Verger M. Psychophysiological stress in tennis players during the first single match of a tournament. Psychoneuroendocrinologyt. 2009;34:150-7.

19. Cohen J. Statistical power analysis for the behavioral sciences. 2nd ed. Hillsdale: Lawrence Earlbaum; 1988.

20. Modrońo C, Guillén F. Anxiety characteristics of competitive windsurfers: relationships with age, gender, and performance outcomes. J Sport Behav. 2011;34:281-94.

21. Dias C, Cruz JF, Fonseca AM. Emoçóes, "stress", ansiedade e "coping”: estudo qualitativo com treinadores de nível internacional. Rev Bras Educ Fís Esporte. 2010;24:331-42.

\section{Agradecimentos}

As autoras agradecem à CAPES pela concessão de bolsa de estudos e a Secretaria de Esporte, Lazer e Juventude de Curitiba (SMELJ), Lei de Incentivo ao Esporte, sob n. 01-0184144/2012.

$$
\begin{array}{r|r}
\text { ENDEREÇO } & \\
\text { Thais do Amaral Machado } & \\
\text { Departamento de Educação Física } & \\
\text { Setor de Ciências Biológicas } & \text { Recebido para publicação: 24/01/2014 } \\
\text { Universidade Federal do Paraná } & \text { 1a. Revisão: 29/11/2014 } \\
\text { R. Coração de Maria, } 92 & \text { 2a. Revisão: 26/01/2015 } \\
\text { 81531-980 - Curitiba - PR - BRASIL } & \text { 3a. Revisão: 10/05/2015 } \\
\text { e-mail: tha.thatha25@gmail.com } & \text { Aceito: 17/07/2015 }
\end{array}
$$

